\title{
Analyse Thématique Des Récits De Vie Des Cadres Épuisés Du Secteur Privé Marocain
}

\author{
Ahmed Maghni, Professeur PES \\ Ikrame El-Abbadi, Doctorante
}

Ecole Nationale de Commerce et de Gestion de Tanger

Université Abdelmalek Essaadi, Maroc

Doi:10.19044/esj.2019.v15n28p22～URL:http://dx.doi.org/10.19044/esj.2019.v15n28p22

Résumé

Cette étude a pour objectif d'appréhender l'épuisement professionnel des cadres dans le secteur privé au Maroc. Plus précisément, il s'agit de révéler les causes de la « souffrance au travail », et d'en comprendre, notamment, les déclencheurs, les manifestations et les déterminants. Pour cela, nous analysons les résultats des récits de vie réalisés auprès de quatorze cadres marocains épuisés. Les résultats de l'analyse thématique des corpus collectés ont permis de dégager les propriétés de l'épuisement professionnel, ses antécédents et ses effets. Ils mettent en évidence également les conditions de travail générant le burnout, particulièrement, le rythme de travail élevé, la charge quantitative de travail, l'imprécision des tâches à effectuer, le manque de reconnaissance et le conflit entre vie professionnelle/vie privée. Ainsi, ils contribuent à comprendre les deux formes de comportements adoptés par les cadres au moment de l'épuisement : les refuges et l'intention de quitter.

Mots clés: Cadres épuisés, Etude exploratoire hybride, Analyse séquentielle, Analyse thématique 


\title{
Thematic Analysis of the Life Stories of the Exhausted Executives in the Moroccan Private Sector
}

\author{
Ahmed Maghni, Professeur PES \\ Ikrame El-Abbadi, Doctorante \\ Ecole Nationale de Commerce et de Gestion de Tanger \\ Université Abdelmalek Essaadi, Maroc
}

\begin{abstract}
This study aims to understand the burnout of executives in the private sector in Morocco. More specifically, it is about revealing the causes of suffering at work, and to understand, in particular, triggers, manifestations and determinants. For this, we analyze the results of life stories from fourteen exhausted Moroccan executives. The results of the thematic analysis of the corpora collected have revealed the properties of burnout, its antecedents and its effects. They also highlight the working conditions generating burnout, particularly the high work rate, the quantitative workload, imprecision of tasks, lack of recognition and conflicts between professional and private life. Thus, they help to understand the two forms of behavior adopted by executives at the time of exhaustion : shelters and the intention to leave.
\end{abstract}

Keywords: Exhausted executives, Hybrid exploratory study, Sequential analysis, Thematic analysis

\section{Introduction}

Des études récentes publiées par l'Organisation de Coopération et de Développement Economique (OCDE, 2012); Technologia (2014); Le baromètre Cegos (2016) révèlent que beaucoup de salariés, notamment dans les économies occidentales, souffrent d'une détérioration de la qualité de vie au travail. Ce constat a fait l'objet de dénonciation non seulement de la part des salariés, mais aussi, de la part des chercheurs, des médecins spécialistes, des associatifs, et d'autres acteurs.

Face aux changements organisationnels perpétuels, l'individu devient de plus en plus vulnérable à des risques d'ordres psychosociaux, notamment, l'épuisement professionnel (EP). L'Organisation Mondiale de la Santé (OMS) estime que le burn-out deviendra, après les maladies cardiovasculaires, la deuxième cause d'invalidité chez l'humain en 2020. Ce mal étrange peut être 
une source d'affaiblissements physiques et d'apparition des troubles émotionnels et mentaux.

Bien que le phénomène ait fait l'objet de plusieurs recherches depuis les années soixante-dix, tant au niveau empirique que théorique, ce n'est qu'en 2004 que le Bureau International de Travail a reconnu l'EP comme un risque psychosocial. Le Maroc, par rapport aux pays développés et au regard des changements qu'a connus le monde professionnel, n'a pas fait recours à des ajustements au niveau de son Code du travail depuis son entrée en vigueur en 2004, notamment, au niveau de la loi marocaine $\mathrm{n}^{\circ} 65-99$. L'un des objectifs cruciaux de cette loi est d'assurer la santé physique et morale des travailleurs marocains. Cependant, le code du travail néglige entièrement la santé psychologique des salariés, y compris l'épuisement professionnel qui n'était même pas mentionné. De plus, il est quasiment impossible de trouver des ouvrages, des thèses ou des articles scientifiques marocains mettant en lumière ce dysfonctionnent au niveau du code de travail. Toutefois, ce phénomène devient de plus en plus relevé par l'opinion publique. Des articles de presse, des émissions télévisées et des acteurs associatifs signalent cette vérité amère vécue par les salariés. Ils encouragent une prise de conscience de la gravité de ce mal-être. De ce fait, nous estimons à travers cette recherche de sensibiliser les individus et mettre en place des programmes de préventions efficaces. De plus, nous souhaitons révéler l'ampleur de ce phénomène, tout en analysant les causes éventuelles de son apparition. Plus précisément, nous désirons répondre à quelques questionnements : Comment les cadres marocains vivent leur épuisement professionnel ? Quels en sont les déclencheurs ? Quelles en sont les causes ? Et comment se manifeste-t-il ?

Pour cela, nous avons effectué quatorze entretiens en respectant le principe de saturation théorique (Glaser \& Strauss, 1967). Ces entrevues ont été menées auprès des cadres épuisés du secteur privé. Nous les avons invités à s'exprimer profondément dans le cadre d'entretiens en face-à-face de type « récit de vie ». Par ailleurs, un travail de recueil, de codage des données et des analyses fréquentielles a été effectué pour évaluer le poids des thèmes prononcés par les cadres.

Nous présentons donc, le cadre conceptuel et théorique de l'épuisement professionnel, avant d'expliquer soigneusement la méthodologie adoptée et les résultats obtenus.

\section{Cadres conceptuel et théorique de l'épuisement professionnel}

Le phénomène de l'épuisement professionnel a connu son essor aux États-Unis dans le milieu des soignants suite à des enquêtes effectuées par le psychiatre et le psychanalyste Herbert Freudenberger (1970). Dans une « Free Clinic » prenant en charge des toxicomanes, l'auteur a observé quelques symptômes sur les soignants bénévoles, tels qu'une fatigue progressive, ainsi 
que des troubles de la conduite et de l'humeur. Ceci l'a amené à utiliser le terme «burn-out» dans ses écrits pour caractériser l'état mental de ces soignants. Par la suite, les recherches se sont étendues aux différents secteurs et professions. Les recherches empiriques se sont élargies pour traiter presque tous les métiers, notamment ceux où les contacts sont moins importants. Selon Schaufeli et Taris (2005), il n'y a pas de raison que le burn-out soit limité au secteur des services humains.

Dès 1976, la chercheuse Maslach a mené des études sur l'usure professionnelle, notamment, dans les champs du médical et de la santé mentale, puis chez les avocats. Ces efforts ont conduit à valider et faire reconnaître le concept d'épuisement professionnel. Par la suite, une multitude d'observations et de définitions ont été avancées pour le concept. Selon les approches, le burnout est conceptualisé tantôt comme un processus et tantôt comme un état (N. Leroy-Frémont, P. Desrumaux \& T. Moundjiegout, 2014). Au cours de leurs recherches sur ce phénomène, Freudenberger et Richelson (1980) arrivent à conclure que le burnout survient suite à un échec ressenti chez un individu désirant atteindre certains objectifs ; cet échec lui entraine une frustration. Dans le même sens, Cherniss (1980) avance que ce déséquilibre survient à la suite d'un choc émotionnel. Il apparaît lorsque la vie professionnelle ne satisfait pas les attentes d'une personne. Maslach et Jackson (1981), quant à eux, définissent le burnout comme un syndrome d'épuisement émotionnel, de dépersonnalisation et de réduction de l'accomplissement personnel. Par ailleurs, Pines et Aronson (1988) stipulent que le burnout est un corollaire des troubles émotionnels issus des situations professionnelles difficiles et pouvant générer un épuisement physique, émotionnel et mental. Enzmann, Schaufeli, Janssen, et Rozeman (1998), affirment que le burnout se manifeste dans un contexte de travail rude où il réside un écart aigu entre les attentes et les efforts de l'individu et les exigences réelles du milieu professionnel. Farber (2000), pour sa part, identifie trois formes de burnout. La première appelée « épuisement » concerne les individus soumettent à une iniquité au travail. La deuxième forme est dite «classique », se rapporte à des individus très engagés à leur travail et cherchant la réalisation de soi, en vue de diminuer la souffrance causée par le stress. Par contre, la troisième forme concerne les individus épuisés travaillant dans un milieu professionnel peu stimulant et monotone. Truchot (2004), de son côté, considère que l'épuisement professionnel est une résultante d'un stress lié à des exigences professionnelles accrues. Cependant, Martiat (2005) souligne qu'il faut dissocier entre le burnout et le stress professionnel. Il considère que l'épuisement professionnel se caractérise par une attitude négative envers le travail et l'entourage professionnel, tandis que le stress peut se manifester chez l'individu dans n'importe qu'elle situation professionnel. En outre, Shirom et Melamed (2006) proposent une notion s'inscrit dans les prolongements de la 
théorie de la préservation des ressources (Hobfoll, 1989,2001). Ces deux auteurs définissent le burnout à travers trois dimensions : l'épuisement physique, l'épuisement émotionnel et l'épuisement cognitif.

Par ailleurs, deux approches théoriques principales fondent la recherche sur l'épuisement professionnel : la première, traite l'épuisement en termes de déséquilibre entre les ressources et les exigences au travail. La deuxième, quant à elle, appréhende l'épuisement professionnel en termes d'épuisement de valeurs motivationnelles (Halbesleben Buckley, 2004). La première approche (Demerouti et al. 2001 ; Karasek, 1979 \& Siegrist, 1998) est de nature pathogénique et postule que le burnout est le résultat néfaste d'un déséquilibre entre les exigences et les ressources au travail. La deuxième approche est de nature salutogénique, elle situe le burnout en rapport avec les sources de santé et de motivation de la personne (Emmanuel Abord de Chatillon, 2009). À cet égard, la théorie de la préservation des ressources (Hobfoll, 1989), postule que le burnout survient quand un individu perd, ou pense perdre, une ou plusieurs ressources qui animent sa motivation et auxquelles il tient (Emmanuel Abord de Chatillon, 2009). Cette approche met en avant l'importance des ressources dont dispose la personne pour faire face aux situations difficiles, et met l'accent sur les facteurs susceptibles de diminuer les exigences au travail. Selon cette théorie, l'objectif principal des salariés est de s'efforcer «d'obtenir, de conserver, de protéger et de promouvoir les choses qu'ils valorisent» (Hobfoll, 2001, p. 341) ; selon Hobfoll (2001), « les choses qu'elles valorisent» correspondent aux ressources.

\section{Échantillon et collecte de données}

La présente recherche est exploratoire, Il était donc évident de laisser parler les cadres «épuisés », le face-à-face prend la forme d'entretien compréhensif pour reprendre Kaufmann (1996).

Pour sélectionner les cadres «épuisés » sujets de la présente étude, il a fallu administrer un test de dépistage. Ainsi, un questionnaire scientifique proposé par Pines, nommé «BMS-10, Burnout Measure Short Version », a permis de mesurer le niveau de burnout des cadres. Nous avons évalué le grade d'épuisement d'une quarantaine de personnes ayant participé à l'étude. Et, nous avons invité, par la suite, uniquement ceux ayant un niveau avéré d'épuisement à s'exprimer profondément dans le cadre d'entretiens en face-àface.

Nous avons mené une phase d'interviews de type « récits de vie » auprès de quatorze cadres épuisés. Cette méthode de collecte d'informations nous a été utile pour identifier les évènements à partir desquels la situation professionnelle des cadres s'est dégradée. Toutefois, et dans le but de cadrer l'entretien ouvert et spontané avec nos interviewés, il était nécessaire de 
focaliser sur les thèmes suivants : présentation de la personne et de sa trajectoire professionnelle, présentation de l'entreprise, description du travail au sein de l'entreprise, description des relations interpersonnelle, et description des conditions psychologiques comportant des risques d'épuisement professionnel. Les participants à notre étude travaillent dans des secteurs d'activités et dans des zones géographiques variés. Le tableau ${ }^{\circ} 1$ cidessous présente les caractéristiques des interviewés.

Tableau 1: Caractéristiques des cadres

\begin{tabular}{|c|c|c|c|c|c|c|c|}
\hline$\frac{\mathscr{d}}{\mathscr{c}}$ & Age & $\begin{array}{l}\text { Niveau } \\
\text { d'étude } \\
\text { s }\end{array}$ & $\begin{array}{l}\text { Poste } \\
\text { occupé }\end{array}$ & $\begin{array}{l}\text { Secteur } \\
\text { d'activité }\end{array}$ & $\begin{array}{c}\text { Lieu de } \\
\text { travail }\end{array}$ & $\begin{array}{c}\text { Ancien } \\
\text { neté } \\
\text { dans le } \\
\text { poste } \\
\text { actuel } \\
\end{array}$ & $\begin{array}{l}\text { Années } \\
\text { d'expéri } \\
\text { ence } \\
\text { Préalabl } \\
\text { es }\end{array}$ \\
\hline 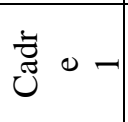 & 30 & $\mathrm{Bac}+5$ & $\begin{array}{c}\text { Responsabl } \\
\text { e d'atelier }\end{array}$ & $\begin{array}{l}\text { Fabrication } \\
\text { et extrusion } \\
\text { du PVT }\end{array}$ & $\begin{array}{c}\text { Casabla } \\
\text { nca }\end{array}$ & 3 ans & 3 \\
\hline 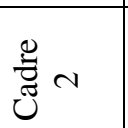 & 35 & $\mathrm{Bac}+5$ & $\begin{array}{l}\text { Directeur de } \\
\text { Succursale }\end{array}$ & Bancaire & $\begin{array}{c}\text { El- } \\
\text { Jadida }\end{array}$ & 4 ans & 8 \\
\hline 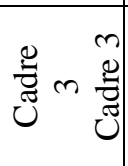 & 29 & $\mathrm{Bac}+5$ & $\begin{array}{l}\text { Responsabl } \\
\text { e des RH et } \\
\text { communicat } \\
\text { ion interne }\end{array}$ & Automobile & $\begin{array}{c}\text { Casabla } \\
\text { nca }\end{array}$ & 1 an & 4 \\
\hline$\underbrace{\mathscr{Z}}_{0}+$ & 26 & $\mathrm{Bac}+5$ & $\begin{array}{c}\text { Responsabl } \\
\text { e de } \\
\text { missions }\end{array}$ & Consulting & $\begin{array}{c}\text { Casabla } \\
\text { nca }\end{array}$ & 1 an & 3 \\
\hline 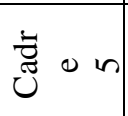 & 39 & $\begin{array}{c}\text { Doctora } \\
\mathrm{t}\end{array}$ & $\begin{array}{l}\text { Manager } \\
\text { (DAF) }\end{array}$ & $\begin{array}{l}\text { Télécommu } \\
\text { nication }\end{array}$ & Tanger & 2 ans & 14 \\
\hline 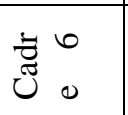 & 32 & $\mathrm{Bac}+5$ & Manager & Automobile & Tanger & 7 mois & 10 \\
\hline$\underset{\tilde{U}}{\tilde{U}} \sim \overline{\frac{0}{0}}$ & 27 & $\mathrm{Bac}+5$ & $\begin{array}{c}\text { Coordinateu } \\
\text { r } \\
\text { d'approvisi } \\
\text { onnement }\end{array}$ & Automobile & Tanger & 2 ans & 1 \\
\hline$\underset{\tilde{U}}{*}, \infty$ & 37 & $\mathrm{Bac}+6$ & $\begin{array}{l}\text { Directeur } \\
\text { d'agence } \\
\text { bancaire }\end{array}$ & Bancaire & Tanger & 10 ans & 6 \\
\hline 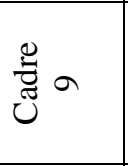 & 31 & $\mathrm{Bac}+5$ & $\begin{array}{l}\text { Responsabl } \\
\text { e de } \\
\text { contrôle de } \\
\text { gestion }\end{array}$ & Automobile & Tanger & 1 an & 4 \\
\hline 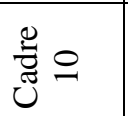 & 36 & $\mathrm{Bac}+5$ & $\begin{array}{c}\text { Cadre } \\
\text { banquier }\end{array}$ & Bancaire & Fès & 10 ans & 1 \\
\hline
\end{tabular}




\begin{tabular}{|c|c|c|c|c|c|c|c|}
\hline 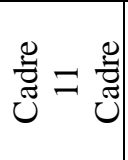 & 24 & $\mathrm{Bac}+5$ & $\begin{array}{c}\text { Responsabl } \\
\text { e d'achat et } \\
\text { d'approvisi } \\
\text { onnement }\end{array}$ & Automobile & Tanger & 8 mois & 0 \\
\hline 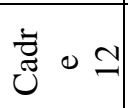 & 42 & $\mathrm{Bac}+5$ & $\begin{array}{c}\text { Cadre } \\
\text { financier }\end{array}$ & Consulting & Fès & 17 ans & 10 \\
\hline 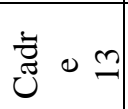 & 27 & $\mathrm{Bac}+5$ & $\begin{array}{c}\text { Cadre } \\
\text { banquier }\end{array}$ & Bancaire & Meknès & 5 ans & 0 \\
\hline 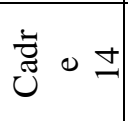 & 24 & $\mathrm{Bac}+5$ & $\begin{array}{c}\text { Ingénieur } \\
\text { de qualité et } \\
\text { client }\end{array}$ & Automobile & Kénitra & 2 ans & 0 \\
\hline
\end{tabular}

\section{Analyse des récits de vie}

Afin de mieux comprendre les stresseurs et les ressources manquantes menant à l'épuisement professionnel chez nos interviewés, deux niveaux d'analyse de discours ont été préconisés : l'analyse intra-cas et l'analyse intercas.

\section{Analyse intra-cas}

Le processus de l'analyse du contenu d'un corpus se caractérise par une progression analytique des données. Les premières étapes servant à organiser les données collectées de façon itérative sont : la codification initiale, le regroupement et la codification thématique.

\section{$\rightarrow$ Analyse séquentielle}

Avant de procéder au traitement des corpus, il était pertinent de réaliser une analyse séquentielle pour les quatorze cas. Elle permet de structurer chronologiquement les événements marquants la trajectoire professionnelle de chaque cadre interviewé, jusqu'à l'apparition de l'événement perturbateur. Pour illustrer les étapes de cette technique d'analyse, nous avons regroupé les événements marquants la vie d'un cadre interviewée dans le tableau n ${ }^{\circ} 2$ ci-dessous :

Tableau 2. Phases de la vie d'un cadre épuisée

\begin{tabular}{|c|c|c|c|}
\hline Séquences & Expressions associées & Évènements & Descriptions \\
\hline S1 & $\begin{array}{l}\text { "Je travaille ça fait à peu près } 4 \text { ans. } \\
\text { Au début j'ai occupé le poste d'analyste } \\
\text { financière. C'était pour } 3 \text { ans ». }\end{array}$ & $\begin{array}{l}\text { L'entrée dans } \\
\text { l'entreprise }\end{array}$ & \multirow{2}{*}{$\begin{array}{l}\text { Évolution de } \\
\text { carrière }\end{array}$} \\
\hline $\mathrm{S} 2$ & $\begin{array}{l}\text { "Après j'ai eu une évolution, une } \\
\text { promotion pour avoir le poste de } \\
\text { responsable contrôle de gestion ». }\end{array}$ & $\begin{array}{l}\text { Promotion } \\
\text { interne }\end{array}$ & \\
\hline S3 & $\begin{array}{c}\text { "Je commence à me sentir mal à l'aise } \\
\text { quand j'ai changé le poste, lorsque j'ai } \\
\text { changé le poste ». } \\
\text { "C'était principalement la nature de } \\
\text { poste, un poste qui a des délais, donc }\end{array}$ & $\begin{array}{l}\text { La rupture- } \\
\text { poste avec } \\
\text { délais }\end{array}$ & $\begin{array}{l}\text { Cadence de } \\
\text { travail très } \\
\text { élevée }\end{array}$ \\
\hline
\end{tabular}




\begin{tabular}{|c|c|c|c|}
\hline & $\begin{array}{c}\text { j'ai des délais hebdomadaires, } \\
\text { journaliers, mensuels, trimestriels... » }\end{array}$ & & \\
\hline S4 & $\begin{array}{c}\text { "Je suis en période de préavis } ; \text { ça fait } \\
\text { deux ans que j'ai déposé ma } \\
\text { démission » }\end{array}$ & $\begin{array}{c}\text { La décision } \\
\text { de départ }\end{array}$ & Démission \\
\hline
\end{tabular}

\section{$\rightarrow$ Codification des données}

La codification des données, quant à elle, représente un processus qui a pour but d'explorer les textes d'interviews. Dans ce travail, trois étapes de codifications ont été menées : la première nommée "codification initiale », qui consiste à affecter des codes (mots ou expressions) à un ensemble de propositions ayant presque le même sens. "Il s'agit simplement de dégager, relever, nommer, résumer, thématiser, presque ligne par ligne, le propos développé à l'intérieur du corpus sur lequel porte l'analyse » (Pierre Paillé, 1994). La deuxième étape est dite « regroupement», permet de réduire le nombre des codes développés dans la première phase de la codification initiale. C'est une méthode par laquelle le chercheur peut nommer, ou affilier un deuxième code regroupant un ensemble de codes initiaux. Ainsi, la «codification thématique» représente la dernière étape de la codification des données, par laquelle le chercheur associe à un regroupement des codes, un concept, une catégorie ou un thème synthétique. Miles et Huberman (2003, p. 133) la définit comme "une façon de regrouper des segments de données en un nombre réduit de thèmes ou d'éléments conceptuels plus synthétiques. ». Le tableau $n^{\circ} 3$ ci-après illustre quelques résultats de la codification thématique.

Tableau 3: Extrait de la codification thématique

\begin{tabular}{|c|c|c|c|}
\hline Expressions associées & Codes & Regroupement & Thème \\
\hline $\begin{array}{l}\text { "Je sors de l'entreprise très fatiguée, très, } \\
\text { très fatiguée ; je ne fais rien, ni ménage, ni } \\
\text { travaux de ménage ni rien, juste je mange, } \\
\text { je fais mes prières et je dors ». }\end{array}$ & $\begin{array}{l}\text { Fatigue } \\
\text { physique }\end{array}$ & \multirow{3}{*}{$\begin{array}{l}\text { Symptômes de } \\
\text { mal-être } \\
\text { physique }\end{array}$} & \multirow{3}{*}{$\begin{array}{l}\text { Épuisement } \\
\text { physique }\end{array}$} \\
\hline $\begin{array}{l}\text { "J'ai commencé à avoir plus de poids, } \\
10 \mathrm{~kg} \text { de plus » }\end{array}$ & $\begin{array}{l}\text { Prise de } \\
\text { poids }\end{array}$ & & \\
\hline $\begin{array}{l}\text { "Et j'ai commencé à avoir des } \\
\text { problèmes ; des douleurs de dos » }\end{array}$ & $\begin{array}{c}\text { Douleurs } \\
\text { de dos }\end{array}$ & & \\
\hline
\end{tabular}

\section{Analyse inter-cas}

Miles et Huberman (2003) confirment que l'utilisation des matrices permet d'ordonnancer les données pour les analyses inter-cas. La segmentation (effectuée précédemment) nous a permis d'aller vers une étape importante, celle de l'analyse thématique. Cette technique facilite l'identification des thèmes abordés et rendre l'analyse des thèmes plus flexibles. Il est utile également pour la formulation des définitions empiriques des thèmes, objet de ce papier. 


\section{$\rightarrow$ Analyse des occurrences}

A ce niveau, nous nous sommes intéressés à calculer la fréquence des thèmes relevés dans l'ensemble des discours considérés comme données. A cet effet, des numéros ont été associés à l'ensemble des thèmes issus des quatorze entretiens pour faciliter le comptage.

Dans cette étape d'analyse, il s'agit de s'intéresser aux énoncés les plus répandus dans le discours des cadres d'entreprises. L'hypothèse est que plus la fréquence d'une idée est élevée, plus cette idée est importante pour le locuteur. Elle correspond au postulat suivant: "l'importance d'une unité d'enregistrement croît avec sa fréquence d'apparition» (Bardin, 1977, p. 140).

Nous avons regroupé le total de tous les thèmes (thèmes, et leurs propriétés), pour déterminer la fréquence de chaque thème dans les quatorze entretiens. Par la suite, nous les avons classifiés, selon leurs estimations obtenues. Le tableau $\mathrm{n}^{\circ} 4$ démontre la classification des thèmes selon leur fréquence d'apparition.

Tableau 4 : Classement des thèmes

\begin{tabular}{|c|l|c|c|}
\hline Classement & \multicolumn{1}{|c|}{ Thèmes } & $\begin{array}{c}\text { Nombre de } \\
\text { répondants }\end{array}$ & Fréquence \\
\hline 1 & Epuisement professionnel & 14 & 28.12 \\
\hline 2 & Conflits & 7 & 15.7 \\
\hline 3 & Isolement & 1 & 13 \\
\hline 4 & Insouciance & 1 & 13 \\
\hline 5 & Manque de reconnaissance & 7 & 12.53 \\
\hline 6 & Charge de travail & 13 & 12.23 \\
\hline 7 & Absence des pratiques RH & 4 & 10.5 \\
\hline 8 & Avenir incertain & 3 & 7 \\
\hline 9 & Conflits de valeurs & 1 & 7 \\
\hline 10 & Déséquilibre VP/vp & 11 & 6.63 \\
\hline 11 & Injustice & 6 & 6.5 \\
\hline 12 & Rythme de travail élevé & 13 & 6 \\
\hline 13 & $\begin{array}{l}\text { Absence de style de } \\
\text { management }\end{array}$ & 5 & 5.4 \\
\hline 14 & Monotonie au travail & 4 & 5 \\
\hline 15 & $\begin{array}{l}\text { Mauvaises conditions } \\
\text { physiques et techniques }\end{array}$ & 2 & 5 \\
\hline 16 & $\begin{array}{l}\text { Normes de travail non- } \\
\text { respectées }\end{array}$ & 4 & 4.75 \\
\hline 19 & $\begin{array}{l}\text { Imprécision des tâches à } \\
\text { effectuer }\end{array}$ & 5 & 3.6 \\
\hline 17 & Intention de quitter & 7 & 3.57 \\
\hline 18 & Cadence de travail élevée & 6 & 3.5 \\
\hline 21 & Absentéisme & 5 & 3 \\
\hline 20 & Manque de soutien & & 1.8 \\
\hline & Non-partage d'informations & & \\
\hline & & & 2 \\
\hline
\end{tabular}




\begin{tabular}{|l|l|c|c|}
\hline 23 & Agressions verbales & 2 & 1 \\
\hline
\end{tabular}

Les résultats obtenus révèlent que les thèmes les plus fréquents sont ceux les plus répétés. "C'est donc la régularité quantitative d'apparition qui est considérée comme significative. Cela suppose que chaque thème a la même valeur. Ce qui n'est pas toujours le cas » (Bardin, 1977, p. 141). A cet effet, Bardin (1977) a proposé une méthode de recalcule permettant l'optimisation des résultats, celle de la pondération.

\section{$\rightarrow$ Fréquence pondérée}

La mesure fréquentielle non pondérée considère que tous les éléments ont une importance égale. Cependant, chaque élément ou thème a son propre poids d'apparition. Pour cette raison, il était crucial de reclasser les thèmes selon leur importance et non seulement selon leur fréquence d'apparition. Dans cette optique, Bardin (1977) a proposé une classification des éléments mesurés selon un système de pondération "Si l'on suppose que l'apparition de tel élément a plus d'importance que tel autre, on peut avoir recours à un système de pondération » (p. 141).

Pour ce faire, nous avons affecté des coefficients aux thèmes considérés importants pour doubler leurs poids. Le tableau $n^{\circ} 5$ reflète cette opération de pondération.

Tableau 5 : Classement des thèmes selon leur fréquence pondérée

\begin{tabular}{|c|l|c|c|c|c|}
\hline Classement & \multicolumn{1}{|c|}{ Thèmes } & $\begin{array}{l}\text { Nombre de } \\
\text { répondants }\end{array}$ & Fréquence & Pondération & $\begin{array}{c}\text { Fréquence } \\
\text { pondérée }\end{array}$ \\
\hline 1 & $\begin{array}{l}\text { Epuisement } \\
\text { professionnel }\end{array}$ & 14 & 28.12 & 2 & 56.24 \\
\hline 2 & Conflits & 7 & 15.7 & 2 & 31.4 \\
\hline 3 & $\begin{array}{l}\text { Manque de } \\
\text { reconnaissance }\end{array}$ & 7 & 12.53 & 2 & 25.06 \\
\hline 4 & $\begin{array}{l}\text { Charge de } \\
\text { travail }\end{array}$ & 13 & 12.23 & 2 & 24.46 \\
\hline 5 & $\begin{array}{l}\text { Déséquilibre } \\
\text { VP/vp }\end{array}$ & 11 & 6.63 & 2 & 13.26 \\
\hline 6 & Injustice & 6 & 6.5 & 2 & 13 \\
\hline 7 & Isolement & 1 & 13 & 1 & 13 \\
\hline 8 & Insouciance & 1 & 13 & 1 & 13 \\
\hline 9 & $\begin{array}{l}\text { Rythme de } \\
\text { travail élevé }\end{array}$ & 13 & 6 & 2 & 12 \\
\hline 10 & $\begin{array}{l}\text { Absence de } \\
\text { style de } \\
\text { management }\end{array}$ & 5 & 5.4 & 2 & 10.8 \\
\hline 11 & $\begin{array}{l}\text { Absence des } \\
\text { pratiques RH }\end{array}$ & 4 & 10.5 & 1 & 10.5 \\
\hline 12 & $\begin{array}{l}\text { Monotonie au } \\
\text { travail }\end{array}$ & 4 & 5 & 2 & 10 \\
\hline
\end{tabular}




\begin{tabular}{|c|c|c|c|c|c|}
\hline 13 & $\begin{array}{l}\text { Intention de } \\
\text { quitter }\end{array}$ & 7 & 3.57 & 2 & 7.14 \\
\hline 14 & $\begin{array}{l}\text { Imprécision } \\
\text { des tâches à } \\
\text { effectuer }\end{array}$ & 5 & 3.6 & 2 & 7.2 \\
\hline 15 & $\begin{array}{l}\text { Cadence de } \\
\text { travail élevée }\end{array}$ & 6 & 3.5 & 2 & 7 \\
\hline 16 & $\begin{array}{l}\text { Avenir } \\
\text { incertain }\end{array}$ & 3 & & & \\
\hline 17 & $\begin{array}{l}\text { Conflits de } \\
\text { valeurs }\end{array}$ & 1 & & & \\
\hline 18 & $\begin{array}{l}\text { Mauvaises } \\
\text { conditions } \\
\text { physiqueset } \\
\text { techniques }\end{array}$ & 2 & 5 & 1 & 5 \\
\hline 19 & $\begin{array}{l}\text { Normes de } \\
\text { travail non- } \\
\text { respectées }\end{array}$ & 4 & 4.57 & 1 & 4.75 \\
\hline 20 & $\begin{array}{l}\text { Non-partage } \\
\text { d'informations }\end{array}$ & 5 & 1.8 & 2 & 3.6 \\
\hline 21 & Absentéisme & & -3 & & \\
\hline 22 & $\begin{array}{l}\text { Manque de } \\
\text { soutien }\end{array}$ & 2 & 2 & 1 & \\
\hline 23 & $\begin{array}{l}\text { Agressions } \\
\text { verbales }\end{array}$ & 2 & 1 & & 1 \\
\hline
\end{tabular}

Nous obtenons donc des résultats différents de ceux obtenus par la mesure à fréquence non pondérée. Néanmoins, nous avons décidé de restituer uniquement les thèmes prononcés par quatre interviewés et plus. Ce choix effectué postule que les thèmes prononcés par plusieurs personnes sont les plus importants. De ce fait, huit thèmes ont été éliminés dans cette phase d'analyse.

\section{Discussion des résultats}

Les résultats de cette étude concernent les déclencheurs, les causes et les manifestations de l'épuisement, et enfin les refuges. Ces derniers représentent un nouveau thème qui n'a pas été traité auparavant et qui est apparu à l'issue de notre étude. Nous allons présenter dans cette partie les définitions empiriques des thèmes expliquant réellement le syndrome d'épuisement professionnel. Ils sont considérés importants, les thèmes les plus fréquents et créant le plus de relations.

\section{Épuisement professionnel et ses propriétés}

L'épuisement professionnel est un processus de mal-être physique et psychique, au cours duquel l'individu ne parvient pas ou peu à supporter les 
stresseurs présents dans l'environnement du travail et le manque de ressources nécessaire pour lui motiver. Étant le thème central de cette étude, l'épuisement professionnel regroupe trois sous-thèmes : épuisement physique, épuisement émotionnel et épuisement cognitif.

La première propriété « épuisement physique »se réfère à un état de mal-être physique justifié par une fatigue, une apparition des maladies somatiques (le reflux gastrique, la maladie auto-immune, la sinusite, les maux de tête...) et des troubles de sommeil, etc.

«Je sors de l'entreprise très fatiguée, très, très fatiguée ». (Cadre 9, 31 ans)

L'«épuisement émotionnel» explique un sentiment de vide, de malaise, et de désintérêt total vis-à-vis du travail et des personnes avec lesquelles le cadre est en relation, quels soient des supérieurs, des collègues, des collaborateurs ou clients. Elle regroupe aussi des réactions émotionnelles disproportionnées (des crises de larmes ou de colère soudaines, irritabilité etc.)

«Toujours énervé, je reviens à la maison fâchée, je dois râler». (Cadre 2, 35 ans)

En fin, l'«épuisement cognitif » peut s'expliquer à travers trois dimensions à savoir : le manque de concentration, la stagnation intellectuelle et la rumination mentale.

* Le manque de concentration correspond à des difficultés de se concentrer, de réfléchir ou de penser à des choses, que ce soit au travail ou ailleurs. Il s'agit d'une baisse de l'énergie mentale.

«Au niveau de la gestion des opérations de caisse, si je trouve des erreurs, je me rends stressé, et je perds ma concentration ». (Cadre 13, 27 ans)

* La stagnation intellectuelle correspond à un sentiment de blocage intellectuel chez les cadres après une certaine période passée dans le même milieu professionnel.

«Maintenant, je suis dans la stagnation ; je suis comme ça... Fixe ! Il n'y a pas un apprentissage peut-être, il n'y a pas ». (Cadre 11, 24 ans)

* La rumination mentale correspond au fait de penser et de repenser à des événements liés au travail en dehors des heures de bureau. Ces événements professionnels prennent une place très importante dans l'esprit et consomment de l'énergie mentale de la personne ruminée.

«Je me trouve même hors des heures de travail en train de penser aux nouvelles conquêtes et aux nouveaux clients. Ça veut dire que je suis toujours perplexe à cause du travail, le travail domine tout mon temps. À chaque fois que je me mets au lit je pense à l'objectif, je me dis comment je vais réaliser mon objectif, il me reste combien des dossiers de crédits ou les suivis ». (Cadre 10, 36 ans) 


\section{Déclencheurs de l'épuisement professionnel}

Les cadres ont été soumis à un ensemble d'événements qui ont basculé leur vie professionnelle. L'analyse séquentielle transversale a été utile pour déterminer ces événements. Neuf déclencheurs ont été identifiés dans les quatorze récits : changement du supérieur, évolution dans la carrière, changement du métier, création du syndicat, encadrement et le suivi d'une nouvelle salariée, travail par objectif, accumulations de travail et absence d'évolution dans la carrière.

Les résultats démontrent que les cadres de l'échantillon ont été soumis à des événements conflictuels variés, mais pour la plupart ceux-ci sont liés entre eux.

Ainsi, dans les quatorze récits, trois cadres (3,11 et 13) ont été confrontés à un changement de leurs supérieurs.

«J'étais à l'aise mais le stress a commencé par l'arrivée d'un nouveau directeur, qui n'était pas à la hauteur de nos attentes. [...] Donc, le stress est devenu de plus en plus élevé ». (Cadre 13, 27 ans)

Quant aux cadres (9, 5 et 2), ils ont connu une évolution dans leurs carrières qui a rendu leurs vies professionnelles précaires et contraignantes.

«Le passage à une autre étape de carrière en tant que DAF sur Tanger [...] donc l'activité du DAF, il est un peu large [...] le fait de gérer une entité assez étendue ça crée du stress et de l'épuisement » (cadre 5, 39 ans).

À l'exception des deux cas (cadre 14 et cadre 7) qui ont indiqué clairement dans leurs discours que l'absence de l'évolution dans leurs carrières était à l'origine de la dégradation de leurs situations.

"Je ne suis pas très motivé, en termes d'évolution surtout. Et lorsque je parle d'évolution, je parle bien évidemment de salaire, de responsabilité, donc quelque chose de nouvelle. Lorsque la personne fait la même chose, donc c'est de l'ennui ». (Cadre 14, 24 ans)

Par ailleurs, le changement du métier a généré des difficultés d'adaptation pour les cadres 6 et 8 .

«Je suis nouveau aussi dans le métier, je ne connais pas le métier de l'entreprise encore, [...] il faut tout faire rapidement. Parfois, c'était fait, et parfois mal fait, pas complet ». (Cadre 6, 32 ans)

En outre, la mutation vers une nouvelle ville était l'événement déclencheur d'un sentiment de mal-être pour le cadre 4.

«Il y a la transition de Tanger vers Casa [...] pour m'adapter ça m'a

pris une année très stressante, une année très, très stressante ». (Cadre 4, 26 ans)

Le cadre 1 a expliqué que son état de mal-être a commencé juste après la création du syndicat dans l'entreprise. 
«Il n'y avait pas du syndicat, après un certain temps, ils l'ont créé, car il y avait beaucoup, beaucoup de problèmes [...] Après chaque période, j'avais eu des nouvelles tâches [...] une surcharge vraiment insupportable ». (Cadre 1, 30 ans)

Pour cadre 12, c'est la charge de travail qui a déséquilibré son parcours professionnel.

«[...] Actuellement, je n'avais pas de temps, parce que le travail augmente; les dossiers s'accumulent et les problèmes s'accumulent en parallèle ». (Cadre 12, 42 ans)

Et enfin, le travail par objectif était le souci du dernier cadre 10. Il a déclaré que sa souffrance a été liée à son travail.

"Dans n'importe quel travail, je vais avoir des difficultés bien sûr, c'est une question de hiérarchie et question de l'objectif [...] je répète pour la nième fois le mot de l'objectif pendant cette interview car, je suis toujours censé l'atteindre ». (Cadre 10, 36 ans)

\section{Causes de l'épuisement professionnel}

La dégradation de l'état de santé physique et psychique des cadres peut s'expliquer aussi par la présence des exigences et l'absence des ressources au travail. Autrement dit, les exigences entravent la vie professionnelle du cadre et les ressources qu'il valorise lui manquent. Nous avons identifié les causes principales contribuant au développement de l'épuisement professionnel. Ces dernières correspondent à celles que les cadres de l'échantillon évoquent comme étant importantes.

L'analyse des récits de vie démontre que les facteurs liés au travail $\left(33.58^{1}\right)$ sont les plus évoqués. Les cadres interrogés rencontrent donc des difficultés liées à la charge de travail, le rythme de travail élevé, la cadence de travail élevée, la monotonie au travail et l'imprécision des tâches à effectuer.

$\mathrm{Si}$ les exigences et les ressources liées au travail sont considérées comme importantes, celles correspondant aux facteurs relationnels (28.33) et organisationnels (12.6) ne sont pas moins importantes. Elles ont été sources des états de mal-être chez certains cadres. En outre, le déséquilibre VP/VP, le seul facteur personnel (6.63) est également prononcé par la plupart des interviewés (11/14), ce qui révèle son effet nocif sur la situation de chaque cadre.

\section{$\rightarrow$ Les facteurs liés au travail}

Le thème charge quantitative de travail a été classé le premier dans la liste des thèmes centraux de cette étude. Il se réfère à une inadéquation entre la quantité des tâches à réaliser et le temps disponible pour leur réalisation.

${ }^{1}$ Fréquence relative 
«Ils (les supérieurs) me débordaient avec plus de travail ; on est dans la période fiscale, on n'a pas mal de dossiers à gérer, moi seul, j'ai trente dossiers à gérer pour ces trois mois». (Cadre 4, 26 ans)

En outre, le thème rythme de travail élevé a été prononcé par la quasitotalité des interviewés (13/14). Il est lié à des heures supplémentaires de travail, généralement rattaché à un nombre important des tâches à effectuer.

"J'ai travaillé jusqu'aux 16 heures par jour pour compenser». (Cadre 1, 30 ans)

Par ailleurs, la cadence de travail élevée était une source principale de mal-être pour six cadres.

«J'ai des délais hebdomadaires, journaliers, mensuels, trimestriels, donc, des délais, des délais, toujours, toujours. Qui dit délai, dit stress, stress, stress, un stress continu, un stress hebdomadaire ». (Cadre 9, 31 ans)

La monotonie au travail fait ainsi partie de la même catégorie thématique (Travail). Il se réfère à l'action de faire et de refaire les mêmes tâches quotidiennement.

«Les tâches que j'effectue sont quotidiennes, répétitives, ça tue ». (Cadre 9, 31 ans)

Également et en dernier lieu, l'imprécision des tâches à effectuer a perturbé la vie professionnelle de cinq cadres. C'est un exercice difficile, une manière de travail floue et anarchique.

"On fait tout et on ne sait pas quoi faire, c'est-à-dire, on n'a pas une fiche de priorité pour dire que cette tâche est prioritaire par rapport à celle-ci [...] pour moi, je ne sais pas exactement quelles sont les tâches dédiées pour mon poste ; il n'y a pas une fiche de poste pour dire que cette tâche ça fait partie de mes compétences ou bien ça fait partie de mon champ de réaction ». (Cadre 7, 27 ans)

\section{$\rightarrow$ Les facteurs relationnels}

La deuxième catégorie qualifiée (relations au travail), regroupe trois thèmes : le conflit, le manque de reconnaissance immatérielle et le non-partage d'informations.

Le thème conflit au travail a été évoqué sous plusieurs angles : avec les supérieurs, les collaborateurs, les collègues et les clients. Il s'agit d'une incompatibilité des points de vue en rapport avec la manière de travail et même avec les attentes et les objectifs de chacun.

«Parfois on arrive à faire des conflits par rapport à ce point de salaire, à un certain moment, je lui (le supérieur) disais que personnellement j'ai d'autres projets professionnels que ce travail, si vous voulez, c'est bien, si ne vous voulez pas, c'est votre question, je ne dépends pas de votre salaire ». (Cadre 7, 27 ans) 
De même, le manque de reconnaissance immatérielle a été cité par cinq cadres, qui ont exprimé amèrement l'absence totale de cette facette de motivation. Cette ressource manquante a contribué à la dégradation de l'état psychique et émotionnel de ces interviewés.

«Je voudrais m'estimer heureuse car à la fin d'année mon supérieur me disait : bravo. Or, durant toute l'année et bien qu'il soit content de mon travail, il ne le montrait pas [...] Je dis toujours pourquoi on reçoit des critiques lorsqu'il le faut et un merci, on ne l'entend pas lorsqu'il le faut ». (Cadre 3, 29 ans)

Le non ou le manque de partage des informations tant en interne (avec collègues, supérieurs...) qu'en externe (avec les clients) renvoie à une difficulté d'accès à l'information.

«Il n'y avait même pas qui peux m'expliquer cette matière sert à quoi ; je n'avais pas toutes les informations [...] je n'ai pas accès à tout, il y avait un manque d'informations [...] ». (Cadre 12, 42 ans)

\section{$\rightarrow$ Les facteurs organisationnels}

Cette catégorie regroupe cinq thèmes : le manque de reconnaissance matérielle, l'injustice salariale, le mode de management disproportionné, les normes de travail non-respectées et l'absence des pratiques $\mathrm{RH}$.

Le manque de reconnaissance matérielle est un sentiment d'absence ou de manque de récompense matérielle.

«Je réalise un travail de plus sans être récompensé. Actuellement, j'occupe l'intérim $d u$ directeur sans indemnité, j'ai deux responsabilités : conseiller commercial et directeur. Donc, avec les mêmes indemnités et les mêmes conditions ». (Cadre 10, 36 ans)

En second lieu, le thème injustice salariale désigne un sentiment d'injustice ressenti par le salarié à l'égard de son salaire, et ce en comparaison avec ce que perçoivent des salariés de même formation ou exerçant le même travail, qu'ils soient interne à l'entreprise ou externe.

«Je perçois le même salaire avec une augmentation de $150 \mathrm{dh}$. Ce $n$ 'est rien par rapport aux autres et par rapport aux évolutions du marché. Je fournis plus d'efforts que les autres, tout en recevant un salaire inférieur. Avec le travail que je fournis, mon salaire doit être le triple de ce qu'il est maintenant ». (Cadre 7, 27 ans)

Le mode de management disproportionné, quant à lui, était un des éléments clés de l'apparition de l'épuisement. À travers les verbatims des cadres, l'incompétence des supérieurs, la déshumanisation au travail, l'absence d'un décideur, le manque d'empathie et d'exemplarité de la part des supérieurs sont les caractéristiques d'un mode de management faible.

«[...] Elle (l'entreprise) est devenue une machine qui emploie des gens, qui n'ont aucune relation entre eux. L'essentiel, c'est la réalisation des objectifs. On reçoit quotidiennement l'évaluation par 
rapport à des chiffres, point final. Il n'y a pas l'aspect personnel. Des fois, la veille je reçois une demande de déplacement à Casa ». (Cadre 8,37 ans)

Puis, le thème «normes de travail non- respectées » désigne une absence totale des restrictions légales au sein de l'entreprise. Des exigences obligatoires ne respectent pas les droits de l'homme au travail.

« [...] en fin de mois, il y avait un patron qui voulait faire des trucs, des cavaleries entre les sociétés: des crédits entre sociétés, des déplacements entre sociétés et tout ». (Cadre2, 35ans)

En fin, l'absence des pratiques RH réfère à un manque des pratiques et des règlements permettant une adaptation facile pour les salariés.

« [...] je n'ai pas eu de formation. Normalement, un nouveau candidat doit avoir une formation, doit connaître les gens de département et doit faire une tournée dans l'entreprise, alors, ce n'était pas le cas pour moi. Je me suis mis directement dans le poste ». (Cadre 7, 27ans)

\section{$\rightarrow$ Le facteur personnel}

Cette catégorie rassemble un seul facteur personnel produisant de l'épuisement, il s'agit du thème : déséquilibre entre la vie professionnelle et la vie personnelle (VP/vp) des cadres.

Le déséquilibre entre VP/vp est un clivage entre les deux vies : privée et professionnelle. Généralement, la vie professionnelle accapare la vie personnelle du cadre. Il représente un problème presque pour la majorité des interviewés (11/14).

«Je n'ai pas de vie privée, je n'ai que le boulot (rire), je n'ai qu'une vie professionnelle ». (Cadre 4, 26 ans)

\section{Conséquences de l'épuisement professionnel}

L'étude effectuée a été une occasion non seulement pour révéler les déclencheurs et les causes de l'épuisement, mais également pour identifier ses conséquences. Les résultats dévoilent que la moitié des cadres de l'échantillon annoncent avoir l'intention de quitter leurs structures «Si je trouve un alternatif maintenant, je ne reviens pas à la société demain, même sans préavis. Si je trouve l'opportunité de changer, je vais changer » (Cadre 11, 24 ans). Cependant, trois cadres (1, 3 et 9) ont déposé leurs démissions en vue de trouver une autre vocation professionnelle moins stressante.

\section{Refuges adoptés au moment de l'épuisement}

Les résultats obtenus mettent en lumière les différents refuges prononcés par les cadres pour alléger leur souffrance. Ainsi, six refuges ont été dégagés des discours traités : boire du café noir, parler à des proches (parents, ami(e)s, époux ou épouse), faire du sport, faire des lectures, fumer les cigarettes, et aller vers l'extérieur. 
Ces refuges sont un ensemble de comportements ou des solutions de bricolages adoptés par les cadres pour faire face à l'épuisement. Chaque interviewé déclare recourir à un ou deux refuges.

Par ailleurs, les résultats reflètent la diversité des comportements ou des solutions adoptées par les cadres épuisés. Certains refuges peuvent aider à rétablir la situation des cadres, d'autres refuges, par contre, peuvent l'aggraver, notamment lorsque le refuge se transforme en une addiction. C'est le cas des deux refuges, boire du café noir et fumer les cigarettes.

L'étude a démontré que trois cadres (1, 4, 13) déclarent avoir l'habitude de boire le café noir pour se déstresser.

«Mon refuge, c'est de prendre un café. Au milieu de travail, et en période de déjeuner, je prenais tous les jours un café » (Cadre 1, 30 ans).

Quatre cadres $(2,4,8,9)$ confirment partager avec leurs proches leur souci au travail.

«À part Dieu, qui est l'ultime refuge, je me concerte avec des amis qui sont des experts dans leurs domaines. Donc, tout dépend du problème, tout dépend des circonstances et de la situation du contexte, donc je fais appel à quelqu'un s'il peut m'aider par exemple ». (Cadre 8, 37 ans)

Quatre cadres $(5,6,11,14)$ pratiquent le sport.

«[...] Le sport, c'est l'un des meilleurs remèdes sincèrement. Le dimanche matin, je fais du sport, c'est parmi les meilleurs remèdes pour oublier la semaine et commencer une autre semaine [...]» (cadre 11, 24 ans).

Par ailleurs, trois cadres $(5,7,14)$ aiment la lecture.

«[...] C'était un refuge parmi autres. Faire des études, faire des lectures, c'est bien pour casser la monotonie et permet de faire face au stress ». (Cadre 5, 39 ans)

Trois cadres $(11,12,13)$ préfèrent aller vers l'extérieur et marcher pour vider leurs stress et colères.

«Je sors carrément, je change l'emplacement, c'est un moyen parmi d'autres pour me libérer du stress, et de la colère. Puis, je rentre une autre personne ». (Cadre 13, 27 ans)

Et, un seul déclare qu'il fume souvent après chaque situation conflictuelle.

«Je fume, mais je ne suis pas un grand fumeur, c'est le meilleur refuge après chaque malentendu ou conflit avec n'importe quelle personne ». (Cadre 10, 36 ans)

\section{Conclusion}

Cette recherche est une occasion pour révéler la réalité douloureuse de la vie professionnelle dans le secteur privé marocain. Le burnout dans cette 
présente étude vient de refléter une image parmi d'autres sur la souffrance des salariés au travail. L'ensemble des résultats obtenus permettent de proposer un modèle de compréhension de l'impact des conditions de travail sur l'épuisement professionnel et ses conséquences sur l'individu. Des exigences et des ressources professionnelles (comme par ex. rythme et charge de travail et conflit VP/vp) provoquent de l'épuisement chez le cadre. Cet épuisement le conduit à adopter des comportements pour faire face à ce syndrome (refuges) ou penser à quitter l'entreprise (intention de quitter). Ces résultats sont conformes aux conclusions d'une étude menée auprès des infirmiers tunisiens. Cette étude confirme que le burn-out résulte en général d'une surcharge de travail, des relations interpersonnelles difficiles, l'absence de soutien social, de manque d'autonomie, des responsabilités mal définies et de l'insuffisance de la reconnaissance de leur travail (Galaa Telelaz, 2018).Le constat est alarmant, et les avis des cadres sont inquiétants. Il est donc nécessaire de mettre en œuvre des programmes d'intervention de sensibilisation et de prévention efficace. Notre présente étude s'inscrit dans ce contexte.

Concernant les apports de cette étude, ils peuvent être abordés selon deux points de vue. Le premier s'intéresse à l'apport méthodologique et le deuxième concerne l'apport managérial.

L'apport méthodologique réside dans la pertinence de la démarche méthodologique adoptée ; la méthode de collecte de données, les outils et les techniques d'analyses utilisés et même les données collectées et traitées sont relativement diversifiées.

Pour l'apport managérial, cette étude peut donner des pistes et des plans d'actions aux praticiens. La dégradation de la santé des individus au travail et l'apparition de plusieurs risques tels que le burn-out, est une réalité douloureuse. Ce travail a dévoilé toutes les exigences peuvent entraver la survie des cadres au milieu professionnel. À cet effet, les résultats de cette étude seront une piste de réorganisation. Ils peuvent aider les décideurs à revoir leur style de management, à créer un climat favorable au sein de leurs structures et à bien gérer leurs ressources humaines.

L'éclairage effectué dans ce papier concerne l'analyse thématique des corpus. Alors que la dernière étape dans l'analyse de contenu n'a pas été discutée dans ce papier - est le traitement des résultats. Dans cette phase d'analyse, les résultats sont traités d'une façon à être significatifs et valides. Des opérations statistiques permettent de présenter des tableaux, des diagrammes et des matrices des résultats qui mettent en relief les données apportées par l'analyse. Ainsi, les quatorze entretiens ne reflètent pas d'une manière exhaustive la réalité de la vie professionnelle au Maroc. Il est nécessaire donc de compléter cette étude par une autre confirmatoire plus large afin de tester nos hypothèses et propositions théoriques. 


\section{References:}

1. Bardin, L. (1977). L'analyse de contenu. Paris, France: Presses universitaires de France.

2. CEGOS. (2016). Climat social et qualité de vie au travail. 18ème édition de baromètre de de l'observatoire CEGOS.

3. Cherniss, C. (1980). Professional burnout in human service organizations. Praeger.

4. De Chatillon, E. A., Desmarais, C., \& Guenette, A. M. (2009). Managers français et managers suisses: Fatigués et heureux ou fatigués et peu-heureux? Revue économique et sociale. Bulletin de la Société d'études économiques et sociales, 67(2,), p-51.

5. Demerouti, E., Bakker, A. B., Nachreiner, F., \& Schaufeli, W. B. (2001). The job demands-resources model of burnout. Journal of Applied psychology, 86(3), 499.

6. Enzmann, D., Schaufeli, W. B., Janssen, P., \& Rozeman, A. (1998). Dimensionality and validity of the Burnout Measure. Journal of occupational and organizational psychology, 71(4), 331-351.

7. Farber, B. A. (2000). Treatment strategies for different types of teacher burnout. Journal of Clinical Psychology, 56(5), 675-689.

8. Freudenberger, H. J. (1970). The case of the missing male authority. Journal of religion and health, 9(1), 35-43.

9. Glaser, B., \& Strauss, A. (1967). Grounded theory: The discovery of grounded theory. Sociology the journal of the British sociological association, 12(1), 27-49.

10. Halbesleben, J. R. B., \& Buckley, M. R. (2004). Burnout in Organizational Life. Journal of Management, 30(6), 859-879.

11. Hobfoll, S. (2001). The Influence of Culture, Community, and the Nested-Self in the Stress Process: Advancing Conservation of Resources Theory. Applied Psychology, 50, 337-421.

12. Kaufmann, J.-C. (2016). L’entretien compréhensif-4e éd. Armand Colin.

13. Leroy-Frémont, N., Desrumaux, P., \& Moundjiegout, T. (2014). Les effets des demandes au travail et de la justice organisationnelle sur l'épuisement professionnel: Quels effets médiateurs du soutien social et de l'estime de soi? Pratiques psychologiques, 20(4), 231-248.

14. Malakh-Pines, A., \& Aronson, E. (1988). Career burnout : Causes and cures. Free Press.

15. Maslach, C. (1978). The client role in staff burn-out. Journal of social issues, 34(4), 111-124.

16. Miles, M. B., \& Huberman, A. M. (2003). Analyse des données qualitatives. De Boeck Supérieur. 
17. OCDE., (2012). Mal-être au travail ?: Mythes et réalités sur la santé mentale et l'emploi. Santé mentale et emploi, OCDE Publishing, Paris.

18. Paillé, P. (1994). L'analyse par théorisation ancrée. Cahiers de recherche sociologique, (23), 147-181.

19. Schaufeli, W. B., \& Taris, T. W. (2005). The conceptualization and measurement of burnout : Common ground and worlds apart. Work \& Stress, 19(3), 256-262.

20. Shirom, A., \& Melamed, S. (2006). A comparison of the construct validity of two burnout measures in two groups of professionals. International Journal of Stress Management, 13(2), 176-200.

21. Siegrist, J. (1998). Adverse health effects of effort-reward imbalance at work. Theories of organizational stress, 190-204.

22. TECHNOLOGIA. (2014). Etude clinique et organisationnelle permettant de définir et de quantifier ce qu'on appelle communément le burn out. Source : http://www.technologia.fr/blog/wpcontent/uploads/2014/01/Burn-out-Etudeclinique-et organisationnelle-janvier-2014.pdf.

23. Telelaz, G. (2018). L'efficacite D'un Programme Therapeutique Cognitivo-Comportemental Dans La Diminution Du Burn-Out Chez Les Infirmiers De L'hopital De Sfax (Etude DescriptiveExperimentale). European Scientific Journal, ESJ, 14(15), 139.

24. Truchot, D. (2004). Epuisement professionnel et burnout: Concepts, modèles, interventions. Dunod. 